This item was submitted to Loughborough's Research Repository by the author.

Items in Figshare are protected by copyright, with all rights reserved, unless otherwise indicated.

\title{
Exchange rate regime verification: an alternative method of testing for regime changes
}

\section{PLEASE CITE THE PUBLISHED VERSION}

http://dx.doi.org/10.1016/j.econlet.2011.05.050

PUBLISHER

(C) Elsevier

VERSION

AM (Accepted Manuscript)

LICENCE

CC BY-NC-ND 4.0

\section{REPOSITORY RECORD}

Ahmad, A.H., David I. Harvey, and Eric J. Pentecost. 2019. "Exchange Rate Regime Verification: An Alternative Method of Testing for Regime Changes”. figshare. https://hdl.handle.net/2134/14014. 
This item was submitted to Loughborough's Institutional Repository (https://dspace.lboro.ac.uk/) by the author and is made available under the following Creative Commons Licence conditions.

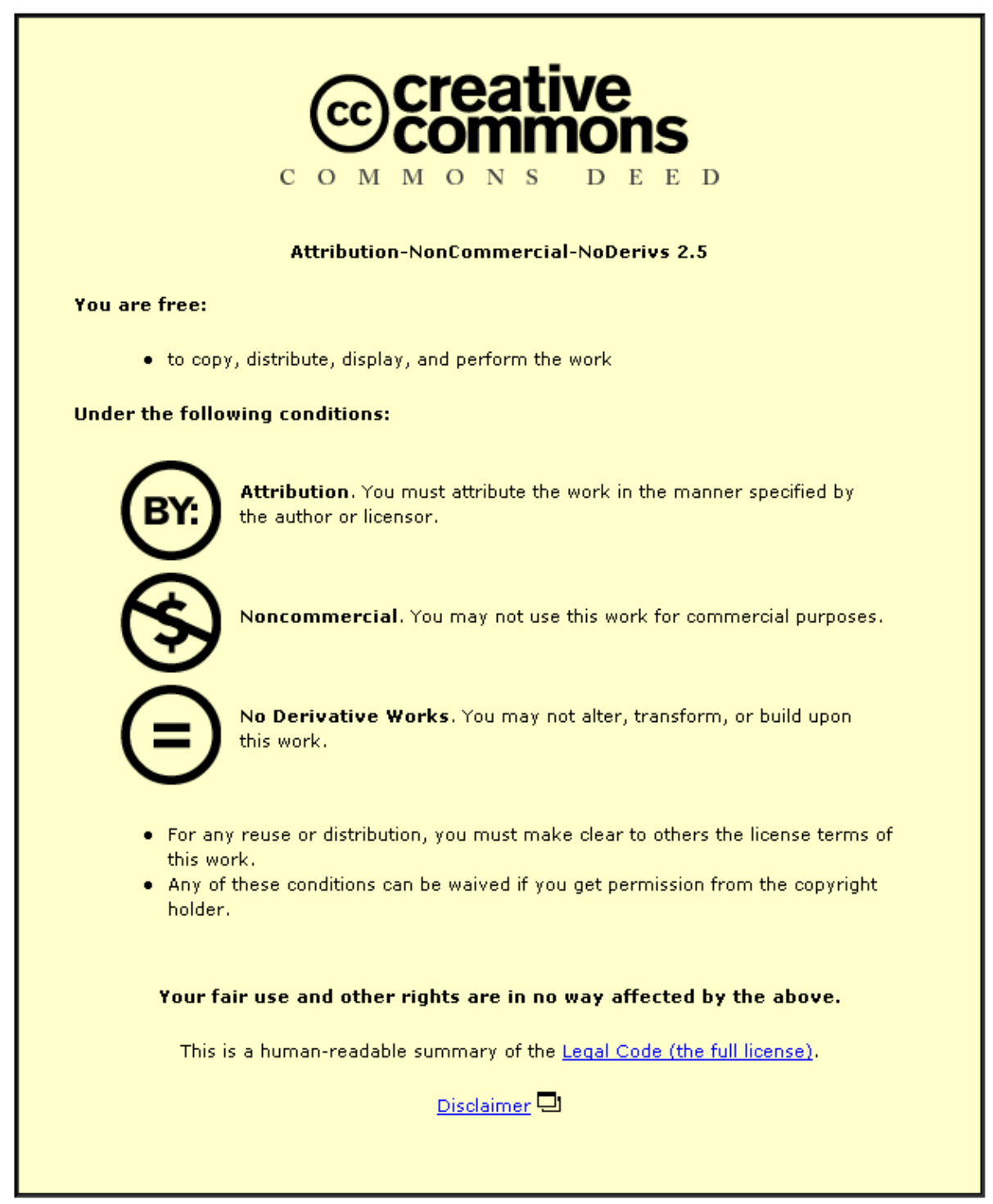

For the full text of this licence, please go to: http://creativecommons.org/licenses/by-nc-nd/2.5/ 


\title{
Exchange Rate Regime Verification: An Alternative Method of Testing for Regime Changes
}

\author{
A. H. Ahmad ${ }^{1}$, David I. Harvey ${ }^{2}$ and Eric J. Pentecost ${ }^{3}$
}

November 2010

\begin{abstract}
This paper proposes a change in persistence test as an alternative method for testing de facto exchange rate regime changes. The tests are applied to 25 African countries, using monthly nominal exchange rate data for the period 1981:012005:12, and the results show that although this approach is broadly complementary to other approaches, it is able to identify some regime changes not picked up by existing methods.
\end{abstract}

\section{JEL Classification numbers: C22, F32}

Keywords: Persistence change tests, de facto exchange rate regime changes

\footnotetext{
${ }^{1}$ Department of Economics, University of Bath, Bath, BA2 7AY, UK. A.H.Ahmad@bath.ac.uk.

${ }^{2}$ School of Economics, University of Nottingham, University Park, Nottingham NG7 2RD, UK. Dave.Harvey@nottingham.ac.uk

${ }^{3}$ Department of Economics, Loughborough University, Loughborough, Leicestershire, LE11 3TU, UK. E.J.Pentecost@lboro.ac.uk.
} 


\section{Introduction}

The seminal paper of Frankel et al (2001) sets out a test to distinguish between announced or official exchange rate regimes and the de facto or practical regime. Alternatively, this paper tests for de facto regime changes using change in persistence tests, following Harvey et al (2006), where the change in an exchange rate regime between fixed and floating can in principle be detected by testing whether a change occurs in the order of integration of the time series. The idea is that a fixed exchange rate regime will be a stationary $\mathrm{I}(0)$ series, while on the other hand, a floating exchange rate regime might show persistence consistent with a non-stationary I(1) series, thus by detecting changes in the order of integration, changes in exchange rate regime can be identified. Our results, based on a sample of 25 African countries, show that the persistence tests can both identify regime changes not identified by other tests and reject regime change where other tests suggest such a regime change. These persistence change tests seem, in general, to be more supportive of the official IMF classification of exchange rate regimes.

The rest of the paper is set out as follows. Section 2 outlines the persistence change tests and Section 3 presents the data and examines the results.

\section{Statistical Tests of Persistence}

A time series $y_{t}, t=1,2, \ldots, T$, may be either $\mathrm{I}(0)$ or $\mathrm{I}(1)$ over all or part of its length. If the series is constant $\mathrm{I}(0)$ or constant $\mathrm{I}(1)$ then the presumption is that there is no change of exchange rate regime. Suppose, however, that a series is $\mathrm{I}(0)$ over the subsample $t=1,2, \ldots, N^{*}$, but $\mathrm{I}(1)$ over the sub-sample $t=N^{*}+1, \ldots, T$; this change in the behaviour of the series may reflect a change in the exchange rate regime. Harvey et al (2006) propose a set of ratio-based statistical tests, which are modified versions of those of Kim (2000), Kim et al (2002) and Busetti and Taylor (2004), and are designed to capture such changes in the persistence of a time series.

Harvey et al (2006) consider the following model for $y_{t}$ :

$$
y_{t}=d_{t}+v_{t}, \quad v_{t}=\rho_{t} v_{t-1}+\varepsilon_{t}
$$

where $d_{t}$ denotes a deterministic component (either a constant or constant plus linear trend), and $\varepsilon_{t}$ is a zero mean process satisfying standard $\alpha$-mixing conditions (e.g. 
stationary ARMA). Under the null hypothesis $H_{0}$ of no change in persistence, $\rho_{t}$ is assumed constant across the full sample period, allowing either constant $\mathrm{I}(0)\left(\rho_{t}=\rho\right.$ $\forall t$ with $|\rho|<1)$ or constant I(1) ( $\left.\rho_{t}=1 \forall t\right)$ behaviour. Under the alternative, the series undergoes a one-time change in persistence, either from $\mathrm{I}(0)$ to $\mathrm{I}(1)$, i.e.:

$$
H_{01}: \rho_{t}= \begin{cases}\rho,|\rho|<1 & t=1, \ldots, N^{*} \\ 1 & t=N^{*}+1, \ldots, T\end{cases}
$$

or from $\mathrm{I}(1)$ to $\mathrm{I}(0)$, i.e.:

$$
H_{10}: \rho_{t}= \begin{cases}1 & t=1, \ldots, N^{*} \\ \rho,|\rho|<1 & t=N^{*}+1, \ldots, T\end{cases}
$$

The true changepoint $N^{*} \in[0.2 T, 0.8 T]$ is assumed unknown, and Harvey et al propose tests based on a sequence of ratio statistics computed at all candidate changepoints:

$$
K_{N}=\frac{(T-N)^{-2} \sum_{t=N+1}^{T}\left(\sum_{i=N+1}^{t} \tilde{v}_{i, N}\right)^{2}}{N^{-2} \sum_{t=1}^{N}\left(\sum_{i=1}^{t} \hat{v}_{i, N}\right)^{2}}
$$

where $\hat{v}_{t, N}$ and $\tilde{v}_{t, N}$ denote the residuals from the OLS regression of $y_{t}$ on $d_{t}$ over the sub-samples $t=1, \ldots, N$, and $t=N+1, \ldots, T$, respectively.

Specifically, three alternative tests are proposed to test $H_{0}$ against $H_{01}$, based on the mean score, mean exponential and maximum principles:

$$
\begin{aligned}
& M S_{m \text { min }}=\exp \left(-b J_{\text {min }}\right)(0.6 T+1)^{-1} \sum_{t=0.2 T}^{0.8 T} K_{t} \\
& M E_{m \text { min }}=\exp \left(-b J_{\text {min }}\right) \ln \left[(0.6 T+1)^{-1} \sum_{t=0.2 T}^{0.8 T} \exp \left(0.5 K_{t}\right)\right] \\
& M X_{m \text { min }}=\exp \left(-b J_{\text {min }}\right) \max _{t \in[0.2 T, 0.8 T]} K_{t}
\end{aligned}
$$

where $\exp \left(-b J_{\min }\right)$ is a Vogelsang (1998)-type correction to ensure that, for a given significance level, the asymptotic null critical values are the same for both constant I(0) and constant I(1) data; see Harvey et al for full details on the computation of $J_{\text {min }}$ and the values of $b$ to be used in each case. Three further tests, denoted by $M S_{m \min }^{\mathrm{R}}$, $M E_{m \text { min }}^{\mathrm{R}}$ and $M X_{m \text { min }}^{\mathrm{R}}$, are proposed for testing $H_{0}$ against $H_{10}$, and these are constructed in the same way as $M S_{m \text { min }}, M E_{m \text { min }}$ and $M X_{m \text { min }}$, respectively, but on replacing $K_{t}$ with $K_{t}^{-1}$ and $J_{\min }$ with an alternative correction statistic $J_{\min }^{R}$. Finally, three tests are also proposed for testing $H_{0}$ against either $H_{01}$ or $H_{10}$; these are 
denoted by $M S_{m \text { min }}^{\mathrm{M}}, M E_{m \text { min }}^{\mathrm{M}}$ and $M X_{m \text { min }}^{\mathrm{M}}$, and are constructed using a combination of information employed in the above tests (see Harvey et al for details). Critical values for all tests are provided by Harvey et al.

A switch from fixed to floating exchange rates should hence be captured by the $M S_{m \min }, M E_{m \min }$ and $M X_{m \min }$ tests; a switch from floating to fixed by a rejection of the null according to the $M S_{m \text { min }}^{\mathrm{R}}, M E_{m \text { min }}^{\mathrm{R}}$ and $M X_{m \text { min }}^{\mathrm{R}}$ tests; and a switch in either direction by $M S_{m \text { min }}^{\mathrm{M}}, M E_{m \text { min }}^{\mathrm{M}}$ and $M X_{m \min }^{\mathrm{M}}$.

\section{Data and results}

We use monthly data on 25 African countries' nominal exchange rates against the SDR for the period 1981 to 2005 giving a sample of 288 observations. Some 14 of these countries were in monetary union arrangements and so there are only 11 nominal exchange rates to consider. Over this time period, 7 of the 13 exchange rates officially changed from a pegged regime to a more flexible regime while the other 6 exchange rates maintained a constant de jure regime, pegged to a basket (Botswana, Morocco), the French franc/Euro (WAEMU, CAEMC) ${ }^{4}$, a crawling peg (Tunisia) or a floating regime (South Africa). Table 1 shows the full list of countries, the de jure exchange rate arrangements over the sample period and in the final column the regime identified by applying the Frankel et al model, which is used for comparison.

Table 2 shows the results of the Harvey et al (2006) modified persistence tests. It confirms a constant level of persistence, i.e. no de facto exchange rate regime change over the sample period, for Botswana, Egypt, Ghana, Morocco, South Africa, Tanzania, Tunisia, Uganda and the CFA Franc zones. There are four countries in this group of ten - Egypt, Ghana, Tanzania and Uganda - where the Harvey et al tests have failed to pick up a de jure regime change, indicating no evidence of a practical regime change for these countries. This is also confirmed by the Frankel et al test, where the pegs in the early part of the period are probably de facto managed floats rather than fixed rate regimes.

For Algeria, Kenya and Nigeria the modified persistence tests show a change in persistence from $\mathrm{I}(0)$ to $\mathrm{I}(1)$, suggesting a switch from a fixed to a more flexible regime over the sample period. This confirms the switch in the de jure regime, but the

\footnotetext{
${ }^{4}$ West and Central African CFA monetary zones, which use CFA franc as their currency.
} 
regimes changes for Algeria and Kenya are not picked up by the test of Frankel et al In these two cases the change in persistence tests concur with the de jure regimes. Furthermore, in the case the CAEMC area, the Frankel et al test identifies a switch from a fixed type of regime to a floating regime, where officially no such switch occurred, while our persistence tests are able to confirm that the exchange rate series for the CAEMC area did not undergo a change in the order of integration suggesting, in line with the IMF's official classification, no change from the announced fixed regime throughout the sample period.

\section{Conclusions}

This paper uses the Harvey et al (2006) modified ratio tests for a change in persistence as an alternative method of testing for changes in de facto exchange rate regimes. Results from our sample of African countries indicate that the tests can give different results from other traditional tests of regime changes of which the model of Frankel et al (2001) is used as the benchmark. Given the difficulty of distinguishing between alternative exchange rate policy regimes, however, it seems most appropriate to regard these tests of persistence change as complementary to other procedures.

\section{References}

Busetti, F. and A. M. R. Taylor (2004) 'Tests of stationarity against a change in persistence', Journal of Econometrics 123, 33-66.

Frankel, J. A., E. Fajnzylber, S. L. Schmukler and L. Serven (2001) 'Verifying Exchange Rate Regimes', Journal of Development Economics 66, 351-386.

Harvey, D. I., S. J. Leybourne and A. M. R. Taylor (2006), 'Modified Tests for a Change in Persistence', Journal of Econometrics 134, 441-469.

Kim, J. (2000) 'Detection of change in persistence of a linear time series', Journal of Econometrics 95, 97-116.

Kim, J., J. Belaire-Franch and R. Badillo Amador (2002) 'Corrigendum to "Detection of change in persistence of a linear time series", Journal of Econometrics 109, 389-392.

Vogelsang, T. J. (1998) 'Trend function hypothesis testing in the presence of serial correlation', Econometrica 66, 123-148. 


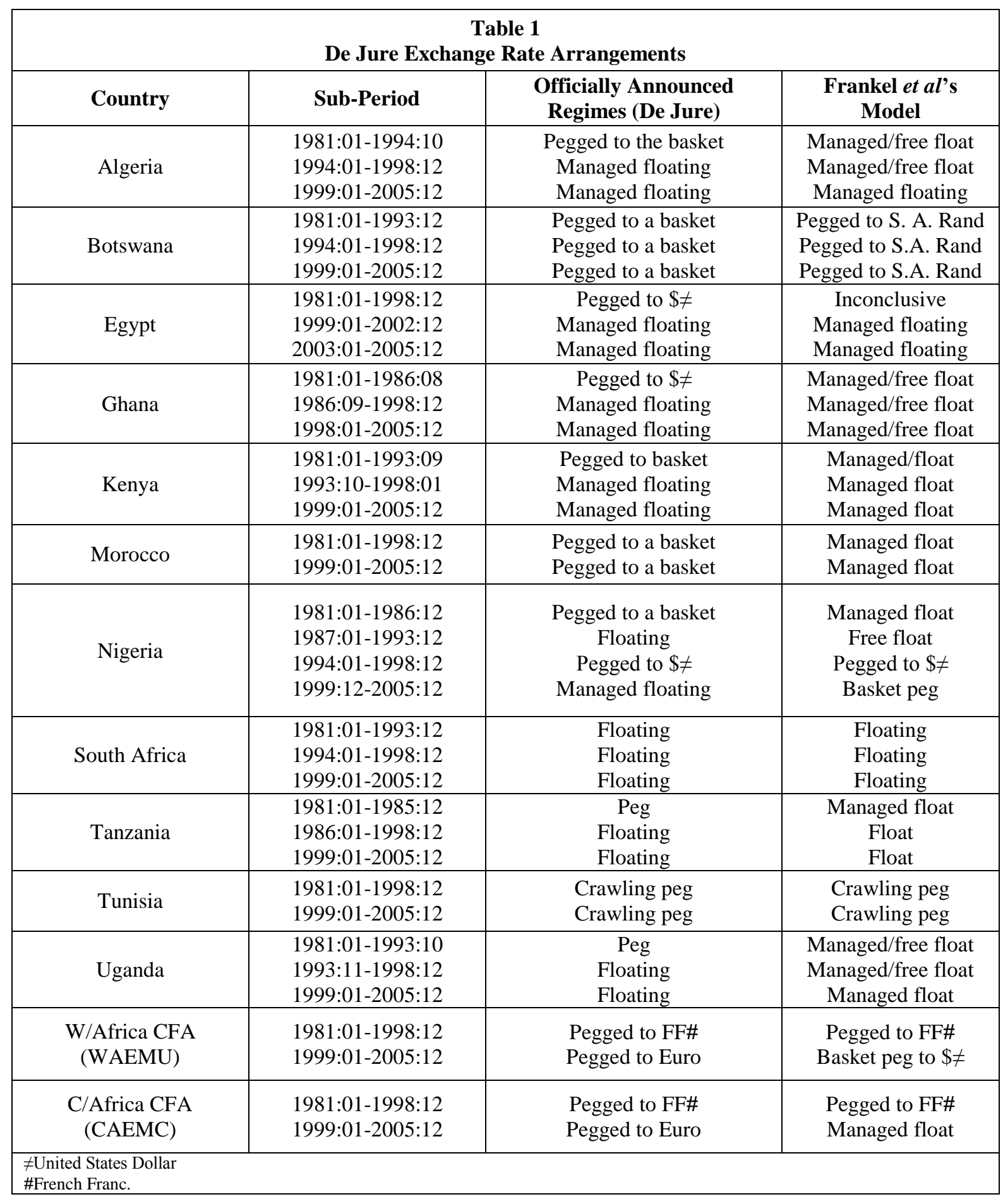




\begin{tabular}{|c|c|c|c|c|c|c|c|c|c|c|}
\hline \multicolumn{11}{|c|}{$\begin{array}{c}\text { Table } 2 \\
\text { Harvey-Leybourne-Taylor Tests for Changes in Persistence }\end{array}$} \\
\hline Country & & $\begin{array}{l}\mathrm{MS}_{\mathrm{m} \min } 10 \% \\
\mathrm{MS}_{\mathrm{m} \min } 5 \%\end{array}$ & $\begin{array}{c}\mathrm{ME}_{\mathrm{m} \min } 10 \% \\
\mathrm{ME}_{\mathrm{m} \min } 5 \%\end{array}$ & $\begin{array}{l}\mathrm{MX}_{\mathrm{m} \min } 10 \% \\
\mathrm{MX}_{\mathrm{m} \min } 5 \%\end{array}$ & $\begin{array}{c}\mathrm{MS}_{\mathrm{m} \min }^{\mathrm{R}} 10 \% \\
\mathrm{MS}_{\mathrm{m} \min }^{\mathrm{R}} 5 \%\end{array}$ & $\begin{array}{c}\mathrm{ME}_{\mathrm{m} \min }^{\mathrm{R}} 10 \% \\
\mathrm{ME}_{\mathrm{m} \min }^{\mathrm{R}} 5 \%\end{array}$ & $\begin{array}{l}\mathrm{MX}^{\mathrm{R}}{ }_{\min } 10 \% \\
\mathrm{MX}^{\mathrm{R}}{ }_{\mathrm{m} \min } 5 \%\end{array}$ & $\begin{array}{l}\mathrm{MS}^{\mathrm{M}}{ }_{\mathrm{m} \min } 10 \% \\
\mathrm{MS}^{\mathrm{M}}{ }_{\mathrm{m} \text { min }} 5 \%\end{array}$ & $\begin{array}{l}\mathrm{ME}^{\mathrm{M}}{ }_{\mathrm{m} \min } 10 \% \\
\mathrm{ME}_{\mathrm{m} \min }^{\mathrm{M}} 5 \%\end{array}$ & $\begin{array}{l}\mathrm{MX}^{\mathrm{M}}{ }_{\mathrm{m} \min } 10 \% \\
\mathrm{MX}_{\mathrm{m} \min }^{\mathrm{M}} 5 \%\end{array}$ \\
\hline \multirow{2}{*}{ Algeria } & Mean Case & $\begin{array}{c}225.35^{*} \\
30.73^{*} \\
\end{array}$ & $\begin{array}{c}161.66^{*} \\
10.84^{*} \\
\end{array}$ & $\begin{array}{c}145.66 \\
94.70 \\
\end{array}$ & $\begin{array}{l}0.00 \\
0.00 \\
\end{array}$ & $\begin{array}{l}0.00 \\
0.00 \\
\end{array}$ & $\begin{array}{l}0.00 \\
0.00 \\
\end{array}$ & $\begin{array}{c}26.84^{*} \\
2.05 \\
\end{array}$ & $\begin{array}{l}9.10^{*} \\
0.35\end{array}$ & $\begin{array}{c}82.21^{*} \\
3.70 \\
\end{array}$ \\
\hline & Trend Case & $\begin{array}{l}1.97 \\
0.15\end{array}$ & $\begin{array}{l}0.04 \\
0.00\end{array}$ & $\begin{array}{l}1.04 \\
0.04\end{array}$ & $\begin{array}{l}0.10 \\
0.01\end{array}$ & $\begin{array}{l}0.00 \\
0.00\end{array}$ & $\begin{array}{l}0.06 \\
0.00\end{array}$ & $\begin{array}{l}0.18 \\
0.01\end{array}$ & $\begin{array}{l}0.00 \\
0.00\end{array}$ & $\begin{array}{l}0.05 \\
0.00\end{array}$ \\
\hline \multirow{2}{*}{ Botswana } & Mean Case & $\begin{array}{l}0.00 \\
0.00\end{array}$ & $\begin{array}{l}0.00 \\
0.00\end{array}$ & $\begin{array}{l}0.00 \\
0.00\end{array}$ & $\begin{array}{l}0.05 \\
0.00\end{array}$ & $\begin{array}{l}0.01 \\
0.00\end{array}$ & $\begin{array}{l}0.09 \\
0.00\end{array}$ & $\begin{array}{l}0.00 \\
0.00\end{array}$ & $\begin{array}{l}0.00 \\
0.00\end{array}$ & $\begin{array}{l}0.00 \\
0.00\end{array}$ \\
\hline & Trend Case & $\begin{array}{l}0.43 \\
0.10\end{array}$ & $\begin{array}{l}0.02 \\
0.00\end{array}$ & $\begin{array}{l}0.20 \\
0.03\end{array}$ & $\begin{array}{l}0.00 \\
0.00\end{array}$ & $\begin{array}{l}0.00 \\
0.00\end{array}$ & $\begin{array}{l}0.00 \\
0.00\end{array}$ & $\begin{array}{l}0.09 \\
0.02\end{array}$ & $\begin{array}{l}0.00 \\
0.00\end{array}$ & $\begin{array}{l}0.03 \\
0.00\end{array}$ \\
\hline \multirow{2}{*}{ Egypt } & Mean Case & $\begin{array}{l}1.05 \\
0.03\end{array}$ & $\begin{array}{l}0.08 \\
0.00\end{array}$ & $\begin{array}{l}1.32 \\
0.02\end{array}$ & $\begin{array}{l}0.00 \\
0.00\end{array}$ & $\begin{array}{l}0.00 \\
0.00\end{array}$ & $\begin{array}{l}0.00 \\
0.00\end{array}$ & $\begin{array}{l}0.02 \\
0.00\end{array}$ & $\begin{array}{l}0.00 \\
0.00\end{array}$ & $\begin{array}{l}0.01 \\
0.00\end{array}$ \\
\hline & Trend Case & $\begin{array}{l}0.05 \\
0.00\end{array}$ & $\begin{array}{l}0.00 \\
0.00\end{array}$ & $\begin{array}{l}0.04 \\
0.00\end{array}$ & $\begin{array}{l}0.00 \\
0.00\end{array}$ & $\begin{array}{l}0.00 \\
0.00\end{array}$ & $\begin{array}{l}0.00 \\
0.00\end{array}$ & $\begin{array}{l}0.00 \\
0.00\end{array}$ & $\begin{array}{l}0.00 \\
0.00\end{array}$ & $\begin{array}{l}0.00 \\
0.00\end{array}$ \\
\hline \multirow{2}{*}{ Ghana } & Mean Case & $\begin{array}{l}0.00 \\
0.00 \\
\end{array}$ & $\begin{array}{l}0.00 \\
0.00\end{array}$ & $\begin{array}{l}0.00 \\
0.00 \\
\end{array}$ & $\begin{array}{l}0.00 \\
0.00\end{array}$ & $\begin{array}{l}0.00 \\
0.00 \\
\end{array}$ & $\begin{array}{l}0.00 \\
0.00 \\
\end{array}$ & $\begin{array}{l}0.00 \\
0.00\end{array}$ & $\begin{array}{l}0.00 \\
0.00 \\
\end{array}$ & $\begin{array}{l}0.00 \\
0.00 \\
\end{array}$ \\
\hline & Trend Case & $\begin{array}{l}0.13 \\
0.00\end{array}$ & $\begin{array}{l}0.00 \\
0.00\end{array}$ & $\begin{array}{l}0.02 \\
0.00\end{array}$ & $\begin{array}{l}0.00 \\
0.00\end{array}$ & $\begin{array}{l}0.00 \\
0.00\end{array}$ & $\begin{array}{l}0.00 \\
0.00\end{array}$ & $\begin{array}{l}0.00 \\
0.00\end{array}$ & $\begin{array}{l}0.00 \\
0.00\end{array}$ & $\begin{array}{l}0.00 \\
0.00\end{array}$ \\
\hline \multirow{2}{*}{ Kenya } & Mean Case & $\begin{array}{l}0.00 \\
0.00\end{array}$ & $\begin{array}{l}0.00 \\
0.00\end{array}$ & $\begin{array}{l}0.00 \\
0.00\end{array}$ & $\begin{array}{l}0.01 \\
0.00\end{array}$ & $\begin{array}{l}0.00 \\
0.00\end{array}$ & $\begin{array}{l}0.01 \\
0.00\end{array}$ & $\begin{array}{l}0.00 \\
0.00\end{array}$ & $\begin{array}{l}0.00 \\
0.00\end{array}$ & $\begin{array}{l}0.00 \\
0.00\end{array}$ \\
\hline & Trend Case & $\begin{array}{l}15.23^{*} \\
4.56^{*}\end{array}$ & $\begin{array}{l}6.57^{*} \\
0.97\end{array}$ & $\begin{array}{l}43.45^{*} \\
8.86^{*}\end{array}$ & $\begin{array}{l}1.26 \\
0.45\end{array}$ & $\begin{array}{l}0.20 \\
0.04\end{array}$ & $\begin{array}{l}1.32 \\
0.36\end{array}$ & $\begin{array}{l}7.81^{*} \\
2.31\end{array}$ & $\begin{array}{l}2.51^{*} \\
0.35\end{array}$ & $\begin{array}{c}19.13^{*} \\
3.50\end{array}$ \\
\hline \multirow{2}{*}{ Morocco } & Mean Case & $\begin{array}{l}0.00 \\
0.00\end{array}$ & $\begin{array}{l}0.00 \\
0.00\end{array}$ & $\begin{array}{l}0.00 \\
0.00\end{array}$ & $\begin{array}{l}0.00 \\
0.00\end{array}$ & $\begin{array}{l}0.00 \\
0.00\end{array}$ & $\begin{array}{l}0.00 \\
0.00\end{array}$ & $\begin{array}{l}0.00 \\
0.00\end{array}$ & $\begin{array}{l}0.00 \\
0.00\end{array}$ & $\begin{array}{l}0.00 \\
0.00\end{array}$ \\
\hline & Trend Case & $\begin{array}{l}0.00 \\
0.00\end{array}$ & $\begin{array}{l}0.00 \\
0.00\end{array}$ & $\begin{array}{l}0.00 \\
0.00\end{array}$ & $\begin{array}{l}0.00 \\
0.00\end{array}$ & $\begin{array}{l}0.00 \\
0.00\end{array}$ & $\begin{array}{l}0.00 \\
0.00\end{array}$ & $\begin{array}{l}0.00 \\
0.00\end{array}$ & $\begin{array}{l}0.00 \\
0.00\end{array}$ & $\begin{array}{l}0.00 \\
0.00\end{array}$ \\
\hline \multirow{2}{*}{ Nigeria } & Mean Case & $\begin{array}{l}7.66 \\
0.09 \\
\end{array}$ & $\begin{array}{l}0.88 \\
0.00 \\
\end{array}$ & $\begin{array}{c}24.82 \\
0.11 \\
\end{array}$ & $\begin{array}{l}0.00 \\
0.00 \\
\end{array}$ & $\begin{array}{l}0.00 \\
0.00 \\
\end{array}$ & $\begin{array}{l}0.00 \\
0.00 \\
\end{array}$ & $\begin{array}{l}0.06 \\
0.00 \\
\end{array}$ & $\begin{array}{l}0.00 \\
0.00 \\
\end{array}$ & $\begin{array}{l}0.08 \\
0.00 \\
\end{array}$ \\
\hline & Trend Case & $\begin{array}{l}370.69^{*} \\
189.53^{*}\end{array}$ & $\begin{array}{l}925.58^{*} \\
601.47^{*}\end{array}$ & $\begin{array}{l}957.09^{*} \\
688.59^{*}\end{array}$ & $\begin{array}{l}0.00 \\
0.00\end{array}$ & $\begin{array}{l}0.00 \\
0.00\end{array}$ & $\begin{array}{l}0.00 \\
0.00\end{array}$ & $\begin{array}{l}819.29^{*} \\
299.98^{*}\end{array}$ & $\begin{array}{l}195.37^{*} \\
171.55^{*}\end{array}$ & $\begin{array}{l}152.96^{*} \\
108.90^{*}\end{array}$ \\
\hline \multirow{2}{*}{ South Africa } & Mean Case & $\begin{array}{l}0.00 \\
0.00\end{array}$ & $\begin{array}{l}0.00 \\
0.00\end{array}$ & $\begin{array}{l}0.00 \\
0.00\end{array}$ & $\begin{array}{l}0.00 \\
0.00\end{array}$ & $\begin{array}{l}0.00 \\
0.00\end{array}$ & $\begin{array}{l}0.00 \\
0.00\end{array}$ & $\begin{array}{l}0.00 \\
0.00\end{array}$ & $\begin{array}{l}0.00 \\
0.00\end{array}$ & $\begin{array}{l}0.00 \\
0.00\end{array}$ \\
\hline & Trend Case & $\begin{array}{l}2.85 \\
0.76\end{array}$ & $\begin{array}{l}0.24 \\
0.03\end{array}$ & $\begin{array}{l}1.86 \\
0.33\end{array}$ & $\begin{array}{l}0.00 \\
0.00\end{array}$ & $\begin{array}{l}0.00 \\
0.00\end{array}$ & $\begin{array}{l}0.00 \\
0.00\end{array}$ & $\begin{array}{l}0.70 \\
0.15\end{array}$ & $\begin{array}{l}0.03 \\
0.00\end{array}$ & $\begin{array}{l}0.30 \\
0.03\end{array}$ \\
\hline \multirow{2}{*}{ Tanzania } & Mean Case & $\begin{array}{l}1.82 \\
0.21\end{array}$ & $\begin{array}{l}0.34 \\
0.01\end{array}$ & $\begin{array}{l}5.73 \\
0.33\end{array}$ & $\begin{array}{l}0.01 \\
0.00\end{array}$ & $\begin{array}{l}0.00 \\
0.00\end{array}$ & $\begin{array}{l}0.00 \\
0.00\end{array}$ & $\begin{array}{l}0.18 \\
0.01\end{array}$ & $\begin{array}{l}0.01 \\
0.00\end{array}$ & $\begin{array}{l}0.28 \\
0.01\end{array}$ \\
\hline & Trend Case & $\begin{array}{l}0.00 \\
0.00 \\
\end{array}$ & $\begin{array}{l}0.00 \\
0.00 \\
\end{array}$ & $\begin{array}{l}0.00 \\
0.00\end{array}$ & $\begin{array}{l}0.00 \\
0.00 \\
\end{array}$ & $\begin{array}{l}0.00 \\
0.00\end{array}$ & $\begin{array}{l}0.00 \\
0.00\end{array}$ & $\begin{array}{l}0.00 \\
0.00 \\
\end{array}$ & $\begin{array}{l}0.00 \\
0.00\end{array}$ & $\begin{array}{l}0.00 \\
0.00\end{array}$ \\
\hline \multirow{2}{*}{ Tunisia } & Mean Case & $\begin{array}{l}0.00 \\
0.00\end{array}$ & $\begin{array}{l}0.00 \\
0.00\end{array}$ & $\begin{array}{l}0.05 \\
0.00\end{array}$ & $\begin{array}{l}0.01 \\
0.00\end{array}$ & $\begin{array}{l}0.11 \\
0.00\end{array}$ & $\begin{array}{l}0.00 \\
0.00\end{array}$ & $\begin{array}{l}0.00 \\
0.00\end{array}$ & $\begin{array}{l}0.00 \\
0.00\end{array}$ & $\begin{array}{l}0.00 \\
0.00\end{array}$ \\
\hline & Trend Case & $\begin{array}{l}0.17 \\
0.05\end{array}$ & $\begin{array}{l}0.04 \\
0.01\end{array}$ & $\begin{array}{l}0.45 \\
0.10\end{array}$ & $\begin{array}{l}0.02 \\
0.00\end{array}$ & $\begin{array}{l}0.00 \\
0.00\end{array}$ & $\begin{array}{l}0.02 \\
0.00\end{array}$ & $\begin{array}{l}0.05 \\
0.01\end{array}$ & $\begin{array}{l}0.01 \\
0.00\end{array}$ & $\begin{array}{l}0.09 \\
0.01\end{array}$ \\
\hline \multirow{2}{*}{ Uganda } & Mean Case & $\begin{array}{l}0.66 \\
0.01\end{array}$ & $\begin{array}{l}0.04 \\
0.00 \\
\end{array}$ & $\begin{array}{l}1.47 \\
0.00\end{array}$ & $\begin{array}{l}0.00 \\
0.00 \\
\end{array}$ & $\begin{array}{l}0.00 \\
0.00\end{array}$ & $\begin{array}{l}0.00 \\
0.00\end{array}$ & $\begin{array}{l}0.00 \\
0.00 \\
\end{array}$ & $\begin{array}{l}0.00 \\
0.00\end{array}$ & $\begin{array}{l}0.00 \\
0.00\end{array}$ \\
\hline & Trend Case & $\begin{array}{l}0.22 \\
0.00 \\
\end{array}$ & $\begin{array}{l}0.00 \\
0.00 \\
\end{array}$ & $\begin{array}{l}0.14 \\
0.00 \\
\end{array}$ & $\begin{array}{l}0.02 \\
0.00 \\
\end{array}$ & $\begin{array}{l}0.00 \\
0.00\end{array}$ & $\begin{array}{l}0.00 \\
0.00 \\
\end{array}$ & $\begin{array}{l}0.24 \\
0.01 \\
\end{array}$ & $\begin{array}{l}0.00 \\
0.00\end{array}$ & $\begin{array}{l}0.22 \\
0.00 \\
\end{array}$ \\
\hline \multirow{2}{*}{$\begin{array}{l}\text { C/Africa } \\
\text { CFA } \\
\text { (CAEMC) }\end{array}$} & Mean Case & $\begin{array}{l}1.29 \\
0.25 \\
\end{array}$ & $\begin{array}{l}0.24 \\
0.03 \\
\end{array}$ & $\begin{array}{l}1.33 \\
0.18 \\
\end{array}$ & $\begin{array}{l}0.00 \\
0.00 \\
\end{array}$ & $\begin{array}{l}0.00 \\
0.00\end{array}$ & $\begin{array}{l}0.00 \\
0.00\end{array}$ & $\begin{array}{l}0.22 \\
0.03\end{array}$ & $\begin{array}{l}0.02 \\
0.00\end{array}$ & $\begin{array}{l}0.16 \\
0.01\end{array}$ \\
\hline & Trend Case & $\begin{array}{l}1.29 \\
0.25\end{array}$ & $\begin{array}{l}0.24 \\
0.03\end{array}$ & $\begin{array}{l}1.33 \\
0.18\end{array}$ & $\begin{array}{l}0.00 \\
0.00\end{array}$ & $\begin{array}{l}0.00 \\
0.00\end{array}$ & $\begin{array}{l}0.00 \\
0.00\end{array}$ & $\begin{array}{l}0.22 \\
0.03\end{array}$ & $\begin{array}{l}0.02 \\
0.00\end{array}$ & $\begin{array}{l}0.16 \\
0.01\end{array}$ \\
\hline \multirow{2}{*}{$\begin{array}{l}\text { W/African } \\
\text { CFA } \\
\text { (WAEMU) }\end{array}$} & Mean Case & $\begin{array}{l}1.30 \\
0.25\end{array}$ & $\begin{array}{l}0.24 \\
0.03\end{array}$ & $\begin{array}{l}1.33 \\
0.18\end{array}$ & $\begin{array}{l}0.00 \\
0.00\end{array}$ & $\begin{array}{l}0.00 \\
0.00\end{array}$ & $\begin{array}{l}0.00 \\
0.00\end{array}$ & $\begin{array}{l}0.22 \\
0.03\end{array}$ & $\begin{array}{l}0.02 \\
0.00\end{array}$ & $\begin{array}{l}0.16 \\
0.01\end{array}$ \\
\hline & Trend Case & $\begin{array}{l}0.01 \\
0.00 \\
\end{array}$ & $\begin{array}{l}0.00 \\
0.00 \\
\end{array}$ & $\begin{array}{l}0.01 \\
0.00 \\
\end{array}$ & $\begin{array}{l}0.02 \\
0.00 \\
\end{array}$ & $\begin{array}{l}0.00 \\
0.00\end{array}$ & $\begin{array}{l}0.02 \\
0.00\end{array}$ & $\begin{array}{l}0.00 \\
0.00\end{array}$ & $\begin{array}{l}0.00 \\
0.00\end{array}$ & $\begin{array}{l}0.00 \\
0.00\end{array}$ \\
\hline
\end{tabular}

